\title{
The NSTX Central Instrumentation and Control System *
}

\author{
P. Sichta, J. Dong, G. Oliaro, K. Tindall \\ Princeton Plasma Physics Laboratory, P.O. Box 451, Princeton, New Jersey 08543
}

\begin{abstract}
Earlier this year the National Spherical Torus Experiment (NSTX) at the Princeton Plasma Physics Laboratory achieved "first plasma". The Central Instrumentation \& Control System [1] was used to support plasma operations. Major elements of the system include the Process Control System, Plasma Control System, Network System, Data Acquisition System, and Synchronization System. This paper will focus on the Process Control System. Topics include the architecture, hardware interface, operator interface, data management, and system performance.
\end{abstract}

\section{Introduction}

The Central Instrumentation \& Control System's Process Control System (CPCS) is a distributed system consisting of computers, operator stations, a network, and interfaces to hardware. Its purpose is to provide integrated control of NSTX's "Engineering" subsystems. Some of the control functions are:

- control and monitoring of all subsystems from the main control room and/or remote operating areas,

- $\quad$ acquire, display, and archive digitized waveforms,

- import/export process-control values,

- annunciate process-control alarms,

- high-level control logic and sequencing.

The engineering subsystems which were integrated for first plasma were Vacuum, Gas Injection, Field Coil Power Conversion, and the Synchronization (Facility Clock) system. Some of these systems are controlled at the device level using a programmable logic controller (PLC). The PLC's were configured and programmed by the subsystem engineers. The job of CPCS is to establish a communication interface with the PLC to transfer process values and commands. Conversely, other systems rely on the CPCS to provide the logic, sequencing, and device interfaces.

\section{EPICS}

The CPCS is based upon EPICS, the Experimental Physics Industrial Control System. EPICS is the result of an ongoing international collaboration involving hundreds of contributors, guided by a few "major" sites. The collaboration is the fruition of a USDOE effort to achieve cost-effective control systems for accelerator-physics research projects. Important features of EPICS are its ability to use common computing and network components, industry-standard software design methodologies, and the capability to run on several operating systems. There is a vast amount of detailed information about EPICS available from the internet[2,3]. These resources include overviews of EPICS, user guides for the individual tools, application development guides, and a list server for interactive EPICS inquiries.

Fig. 1 shows the basic EPICS architecture, consisting of an operator interface station (OPI), a host computer, an input/output controller (IOC), and a network.

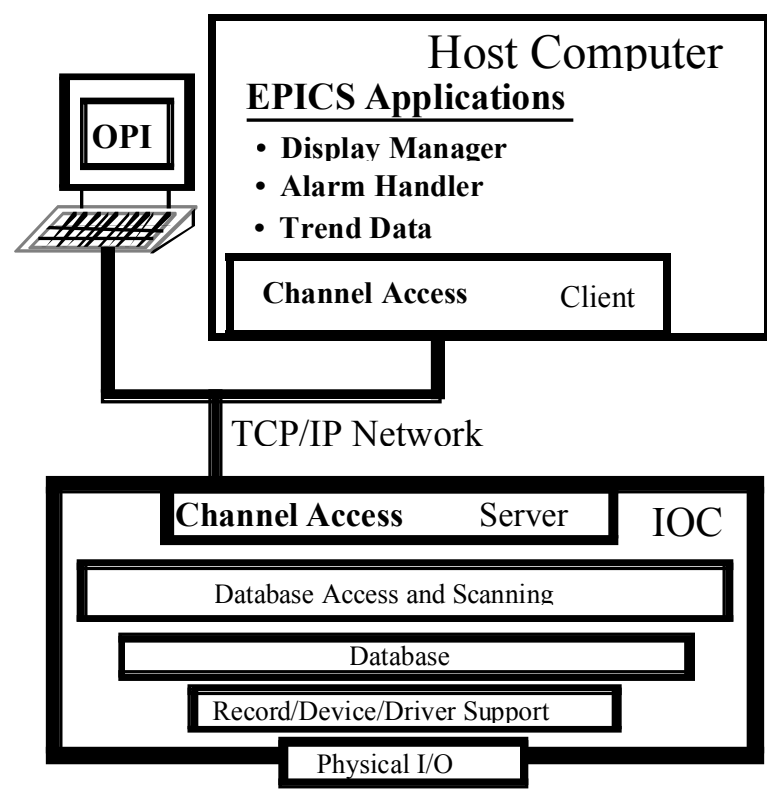

Fig. 1 Basic EPICS Architecture 
The EPICS operator displays and development tools support the X-windows standard. This feature permits networked workstations, X-terminals, and PCs to be used as a hardware platform for system operation and development.

The IOC is the most critical process control component in an EPICS control system. The process-control logic and the device-interface hardware reside in the IOC, allowing it to autonomously control the process. When the IOC is rebooted the control databases are loaded from files on the host computer. The database consists of linked records which perform input, output, calculations, time delays, and other functions. There are about 50 standard record types. Individual sites often create their own record types for special functions. This was done at NSTX to support CAMAC transient digitizers.

The database is developed on the host computer, typically using a point-and-click graphical configuration tool. Using the database configuration tool, a complex process control scheme can be developed without "writing" any code. Of course, in the beginning, learning how to properly configure and connect the records is the challenge.
The host computer serves several functions, none of which are time-critical to the actual process being controlled:

- a boot-server for the IOC,

- an X-window manager for X-terminals/OPI,

- database development tools,

- Operator display editor and manager tools,

- data and source code management.

An Ethernet network connects the IOC and host computer using a communication protocol called Channel Access (CA). CA provides network-transparent access to all IOC databases. When an operator launches a display, the display manager queries the IOC's on the network to see which IOC holds the record for every process variable (PV) or control variable appearing on that display. The IOC's CA server has been designed to significantly reduce network traffic by only transmitting a PV's value to the host/operator's screen when the value has changed.

\section{The NSTX Process Control System}

A block diagram of the NSTX Process Control System is shown on Fig. 2. The hardware platforms and software tools were selected from mature EPICS systems.

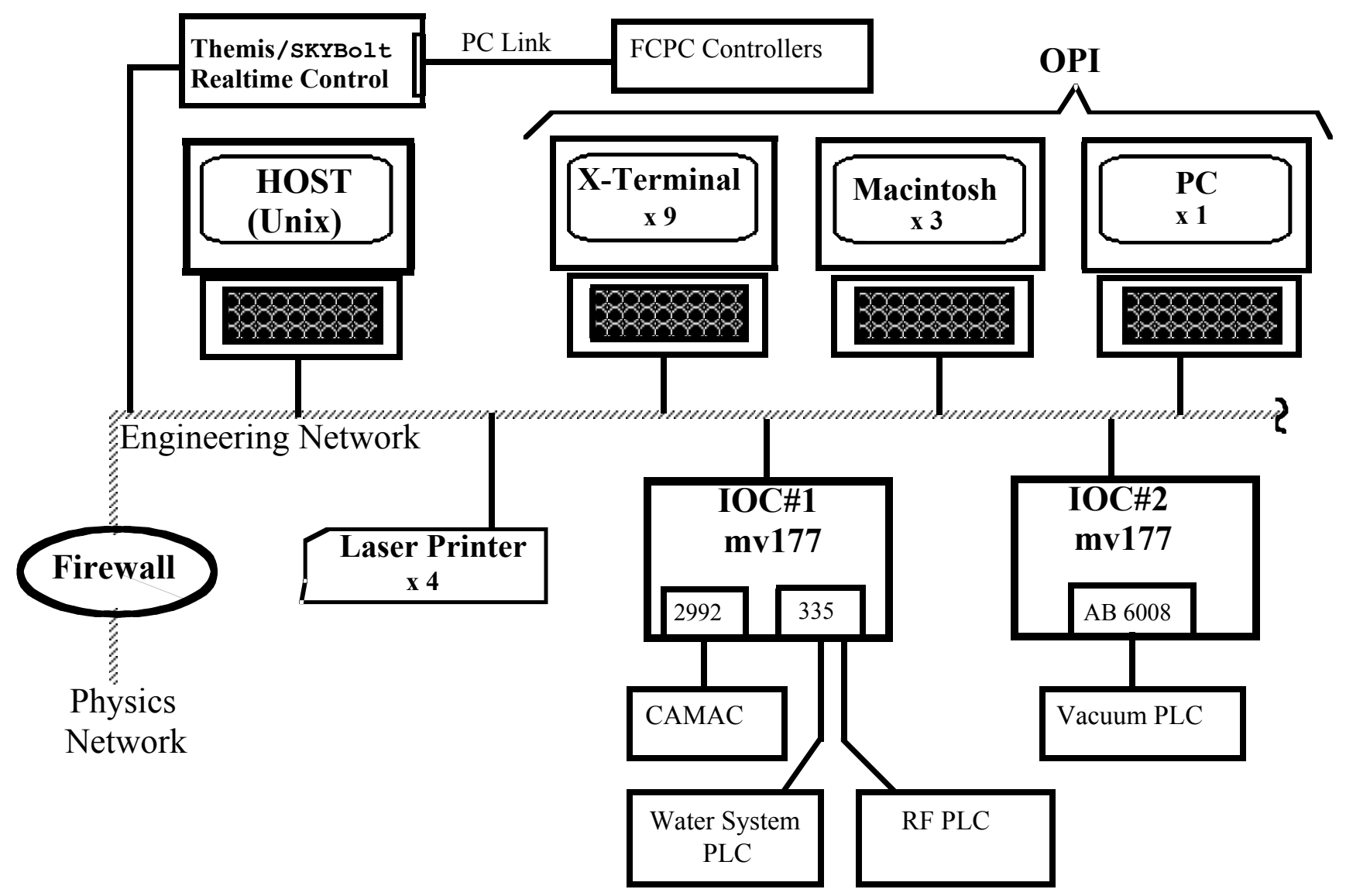

Fig. 2 Block Diagram of the NSTX CI\&C Process Control System 
Even though the older technology hardware we chose offered less performance than the cutting-edge EPICS systems, reliability and proven-performance were of greater importance.

\section{A. $O P I$}

The OPI for NSTX consists of nine X-terminals, three of which are dual-monitor models. In addition, there are four "personal computers" running X-server software. PC's are also used for system development and test. Laptop PCs are used for field testing and integration.

The Motif Editor and Display Manager (MEDM) tool is used for the operator displays. This tool has performed very well. It is easy to use and provides plenty of widgets and other features. About 90 displays are in use at this time. The displays are arranged in a hierarchical manner, providing an intuitive structure for the operators to navigate through. Fig. 3 shows the top-level display.

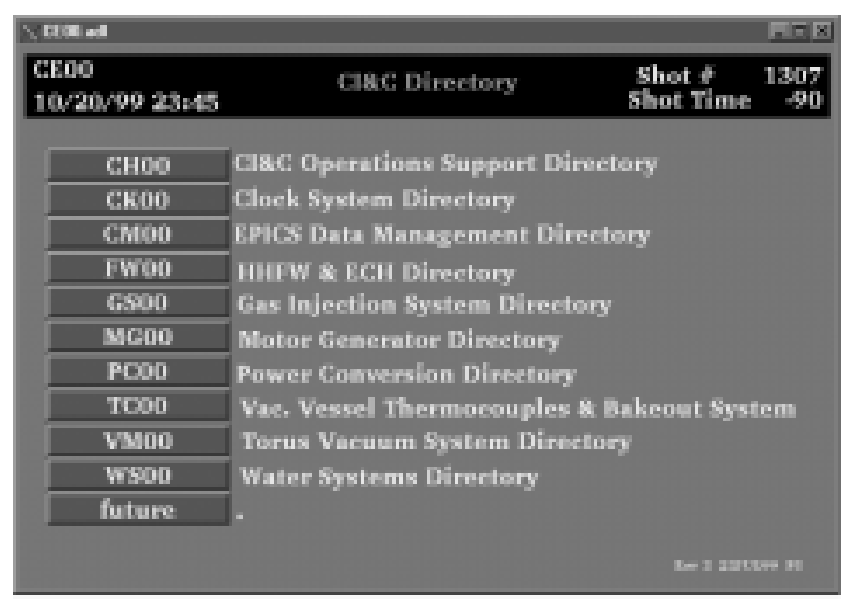

Fig. 3. Top-Level MEDM Display

\section{B. $I O C$}

NSTX required are two IOC's, which are VME-based and include a Motorola 68060-based slot 0 controller (MVME-177) with 32 MB memory. The real-time operating system is VxWorks. In addition to running the control database this CPU runs several "sequencer" programs, written for special-purpose control functions. The IOC typically services about $30 \mathrm{CA}$ connections to client-programs running on the host computer.

NSTX was designed to use legacy I/O hardware from the Tokamak Fusion Test Reactor. This included both CAMAC and PLC equipment. IOC1 has one bit-serial CAMAC link driver. Three brands of PLC's have been integrated, an Allen-Bradley 5/40E, a Gould/784, and a GE Series Six. The 5/40E communicates using A $\mathrm{DCM} /$ scanner configuration from IOC2. The latter two PLC's use the Modbus protocol over serial communication lines from a VME serial module in IOC1. Table I lists some of the significant aspects of IOC1.

Table 1

IOC \& I/O Metrics

\begin{tabular}{|l|c|}
\hline \multicolumn{1}{|c|}{ Parameter } & Value \\
\hline Avg. CPU load & $12 \%$ \\
\hline Peak CPU load & $70 \%$ \\
\hline Avg. Network Load (10 Mb/s port) & $0.6 \%$ \\
\hline \# records & 3000 \\
\hline Avg. record-processing rate (@12\% CPU) & $1000 \mathrm{rec} / \mathrm{s}$ \\
\hline Memory usage (of 32 MB) & $10 \mathrm{MB}$ \\
\hline Number CAMAC points & 500 \\
\hline CAMAC waveform memory & $\sim 6 \mathrm{MB} / \mathrm{shot}$ \\
\hline Number PLC points (total both IOC) & 700 \\
\hline
\end{tabular}

\section{Host Computer}

The host computer is a Sun Ultra 30 workstation, with $256 \mathrm{MB}$ memory. Some of the programs which run on this host are:

- display manager and editor (medm),

- database config. tool (gdct),

- alarm handler (alh),

- waveform plotting (gnuPlot),

- $\quad$ IPCS[5] (data and event import/export).

About a half-dozen programs were written to control special processes in concert with the IOC. These were written in C, using the Channel Access API. Table II lists some performance parameters for the host computer.

Table II.

Host Computer Metrics

\begin{tabular}{|l|c|}
\hline \multicolumn{1}{|c|}{ Parameter } & Value \\
\hline Avg. CPU load & $7 \%$ \\
\hline Peak CPU load & $17 \%$ \\
\hline $\begin{array}{l}\text { Avg. Network Load } \\
(100 \mathrm{Mb} / \mathrm{s} \text { port })\end{array}$ & $1 \%$ \\
\hline
\end{tabular}

A second workstation is available to be used as a backup to the primary host. This will also be used as a test bed for new releases of EPICS and application software, and validating EPICS with new operating system releases.

\section{Network}

Network performance is a critical factor in an EPICS system's performance since all operator commands and display-updates travel over the network. For NSTX, a dedicated "Engineering" network is used for the CPCS. Protecting this network from non-essential traffic and providing protection from unauthorized users is another requirement for the NSTX "Engineering" network. 
A firewall is used to isolate the Engineering network from all other networks at the laboratory. The network uses a switch-based (fiber/copper) topology. Most of the ports are currently running 10 megabit/sec, however, there are a few 100 megabit/sec ports.

\section{Data and Code Management}

NSTX stores all experimental data in an MDSplus data tree [4]. The MDSplus software runs on computers on the NSTX "Physics" network. On the CPCS most of this data originates from CAMAC transient digitizers. After each shot, data requiring permanent archival is read from CAMAC modules (via the IOC) and locally stored on the host computer's disk. A copy is then sent through the firewall to the non-secure network for writing into the tree. The CPCS host computer uses the InterProcess Communication System (IPCS) to notify the MDSplus computer that new data is available. IPCS is also used to import/export data from the IOC, via the host computer.

All engineering subsystems have control databases and operator displays. A few subsystems had special needs which reuquired 'source' code. The code could be IOC sequencer programs, host C programs, or (Unix) scripts. For NSTX a wide, flat application directory structure was chosen. Each engineering subsystem has it's own branch to hold database, source, and operations directories. Insulating the software of the individual subsystems was desireable to enhance code maintenance and subsystem reliability.

\section{Progress Since First Plasma}

The software assembly and startup of EPICS by only two system developers was an difficult task [6]. However, once the basic structure was in place, support for new systems progressed rapidly. Systems which have begun integration in the eight months since "first plasma" are Electron Cyclotron Heating, High Harmonic Fast Wave heating, Coaxial Helicity Injection, Glow Discharge Cleaning, Water Cooling, and Motor Generator.
Some improvements to the "first plasma" configuration of the CPCS are expected :

- $\quad$ programs to support the acquisition and storage of subsystem parameters and control settings, on a per shot basis.

- $\quad$ trending (stripchart) displays and archival of trend data

- a separate computer on the Engineering network dedicated to $\mathrm{X}$-windows management and security enhancement.

\section{Conclusion}

The performance of the CPCS has been a extremely successful. The system was operational for the "first plasma" experimental run, which was achieved 10 weeks ahead of schedule. Hardware and software reliability has been near perfect right from the beginning. The prospects for enhancing the operator's tools to more fully exploit the capabilities of EPICS appear promising. As the control system matures, attention can be focused on targeting engineering resources to enhance specific aspects of the CPCS, such as reliability and maintainability or decreasing post-shot data availability delays.

\section{References}

[1] D. Bashore et al, "Conceptual Design for the NSTX Central Instrumentation and Control System," $17^{\text {th }}$ Symposium on Fusion Engineering, San Diego, CA (1997).

[2] EPICS documents at Argonne National Laboratory, Argonne, $\mathrm{IL}$, available over the internet.

[3] EPICS documents at Los Alamos National Laboratory, Los Alamos, NM, available over the internet.

[4] J.A. STILLERMAN et al., MDSplus Data Acquisition System, 11th Topical Conference on High Temperature Diagnostics (1996).

[5] G. Preckshot, Bill Meyer, et al., "Inter-Process Communication System Programmer Interface Description," unpublished.

[6] P. Sichta, J. Dong, "Startup of the Experimental Physics Industrial Control System at NSTX," $18^{\text {th }}$ Symposium on Fusion Engineering, Albuquerque, NM (1999). 